\title{
Amplify-and-Forward Distributed Beamforming with Local CSI in the Presence of Interferences
}

\author{
Jung-Bin Kim, ${ }^{1,2}$ Ji-Woong Choi, ${ }^{3}$ Hyuk Choi, ${ }^{4}$ and John M. Cioffi ${ }^{1,5}$ \\ ${ }^{1}$ Department of Electrical Engineering, Stanford University, Stanford, CA 94305, USA \\ ${ }^{2}$ Broadcasting \& Telecommunications Media Research Laboratory, ETRI, Daejeon 305-700, Republic of Korea \\ ${ }^{3}$ Department of Information \& Communication Engineering, Daegu Gyeongbuk Institute of Science and Technology (DGIST), \\ Daegu 771-873, Republic of Korea \\ ${ }^{4}$ School of Computer Science, University of Seoul, Seoul 130-743, Republic of Korea \\ ${ }^{5}$ Department of Computer Science, King Abdulaziz University, Jeddah 21589, Saudi Arabia
}

Correspondence should be addressed to Ji-Woong Choi; jwchoi@dgist.ac.kr

Received 13 July 2015; Revised 18 November 2015; Accepted 1 December 2015

Academic Editor: Aline Roumy

Copyright (C) 2015 Jung-Bin Kim et al. This is an open access article distributed under the Creative Commons Attribution License, which permits unrestricted use, distribution, and reproduction in any medium, provided the original work is properly cited.

This paper introduces an optimum amplify-and-forward (AF) distributed beamforming (DBF) in the presence of cochannel interference (CCI) when only local channel-state information (CSI) is available at each relay. It is shown that the proposed DBF closely achieves the performance obtained with global CSI when interference power toward relays is small or there are a large number of interferers but greatly reduces the complexity and overhead. The proposed DBF provides significant improvements over the conventional DBF designed without considering CCI at the cost of slightly increased complexity and overhead and achieves the capacity scaling of $(1 / 2) \log K$ through $K$ relays, where $(1 / 2) \log K$ corresponds to the maximal capacity scaling when there is no CCI.

\section{Introduction}

Cooperative relaying has attracted a great deal of attention because of its appealing properties for both performance and various applications. Among various schemes, cooperative beamforming is being widely considered because it achieves optimal diversity-order performance and capacity scaling by maximizing the received signal-to-noise ratio (SNR). An upper bound on capacity scaling of dual-hop relay networks was provided in [1], in which the capacity scaling was achieved using the consequence of receive and transmit matched filtering at each relay in distributed way. However, an optimum design for beamforming weight was not taken into account in [1]. An optimal distributed beamforming (DBF) to maximize the received SNR was proposed in [2], which showed that the optimal performance is achieved only with local channel-state information (CSI) obtained at each relay. The results of [2] were extended to two-way relaying in [3]; near optimum joint DBF was introduced with which the maximal capacity scaling and full diversity order were achieved.
The above-mentioned works do not consider the impact of cochannel interference (CCI) that is one of the major limiting factors on the performance of wireless communication systems. Recently, [4] introduced optimal beamforming that maximizes the received signal-to-interference-plusnoise ratio (SINR) when $N$ sources perform DBF toward a relay and the destination is corrupted by CCI. However, the impact of CCI was considered only at the destination. Although there is an abundance of research on cooperative beamforming with a variety of scenarios, the distributed approach based on local CSI considering CCI has not yet been thoroughly investigated.

This paper investigates the optimum DBF based on local CSI when the relays and the destination are affected by CCI. The proposed DBF has very small complexity and overhead compared to the cooperative beamforming obtained with global CSI. More details provided in this paper are summarized as follows:

(i) An optimal amplify-and-forward (AF) DBF weight is proposed in the presence of $\mathrm{CCI}$ at both the relays and 
the destination when only local CSI is available at each relay.

(ii) The proposed DBF is shown to achieve nearly the performance obtained with global CSI when there are a large number of interferers or interference power toward relays is small.

(iii) The DBF has a capacity scaling of (1/2) $\log K$ through $K$ relays, where $(1 / 2) \log K$ corresponds to the maximal capacity scaling when there is no CCI.

Numerical results verify that the proposed DBF represents significant improvements over the conventional DBF designed without considering CCI at the cost of slightly increased overhead and complexity.

This paper is organized as follows: Section 2 introduces the system model for DBF protocol. Section 3 presents the optimum DBF weight, and its capacity scaling law is derived. Finally, the numerical results are presented in Section 4, and concluding remarks are given in Section 5.

Notations. $\operatorname{diag}\left[x_{1}, \ldots, x_{K}\right]$ denotes the diagonal square matrix with $x_{1}, \ldots, x_{K}$ on its main diagonal, $(\cdot)^{*}$ the complex conjugate, and $(\cdot)^{H}$ the Hermitian, respectively. $\|\mathbf{a}\|$ is the Euclidean norm of the vector $\mathbf{a}$, and $\mathbf{I}_{K}$ denotes the $K \times K$ identity matrix. $E[X]$ and $\operatorname{VAR}[X]$ mean the expectation and the variance of a random variable (r.v.). $X . \stackrel{\text { w.p.1 }}{\longrightarrow}$ denotes convergence with probability one. For two functions $f(x)$ and $g(x), f(x) \sim g(x)$ means that $\lim _{x \rightarrow \infty} f(x) / g(x)=1$, or equivalently $\lim _{1 / x \rightarrow 0} f(1 / x) / g(1 / x)=1$.

\section{System Model}

Figure 1 depicts a wireless network that consists of a source, a destination, and $K$ relays. Let $\mathcal{S}_{r}=\{1,2, \ldots, K\}$ be a set of the relays. Each node has a single antenna and the relays operate in half-duplex mode with AF strategy. All the relays and the destination are affected by $I$ interferers. Hereafter, subscripts $s, k$, and $d$ denote the source, the $k$ th relay, and the destination, respectively, and $i$ is the index of interferers. Because of the long distance between $s$ and $d$, there is no direct link between them. It is assumed that the activities of interferers change slowly, and, therefore, each node is affected by the same interferers during two phases.

Frequency-flat block-fading channels are assumed, where $h_{i, j}$ denotes the channel coefficient between node $i$ and node $j\left(i, j \in\left\{s, \mathcal{S}_{r}, d\right\}\right)$ and $g_{m, n}$ is the channel coefficient between the $m$ th interferer and receiving node $n$ ( $n \in$ $\left.\left\{\mathcal{S}_{r}, d\right\}\right)$. Channel reciprocity is assumed and each node has the receivers' CSI. The channel coefficients are modelled by independent but not identically distributed (i.n.i.d.) complex Gaussian r.v.s. That is, channel powers $\left|h_{s, k}\right|^{2},\left|h_{k, d}\right|^{2},\left|g_{i, k}\right|^{2}$, and $\left|g_{i, d}\right|^{2}$ are independent and exponentially distributed r.v.'s whose means are $\sigma_{s, k}^{2}, \sigma_{k, d}^{2}, \sigma_{I, i, k}^{2}$, and $\sigma_{I, i, d}^{2}$, respectively.

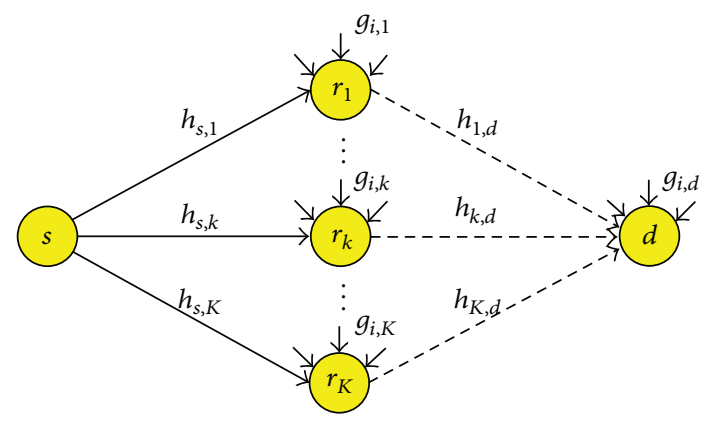

FIGURE 1: System model. A source communicates with a destination through $K$ intermediate relays, where the relays and the destination are affected by a number of $I$ interferers. The relays operate in a halfduplex mode with AF strategy.

During the first phase, $s$ transmits $x_{s}$ with power $P_{s}$. The received signal at relay $k$ is corrupted by multiple interfering signals $x_{i, 1}$ 's with power $P_{i}$ 's:

$$
y_{k}=h_{s, k} \sqrt{P_{s}} x_{s}+\sum_{i=1}^{I} g_{i, k} \sqrt{P_{i}} x_{i, 1}+n_{k} \text {, }
$$

where $n_{k} \sim \mathcal{N}_{c}(0,1)$ is complex additive white Gaussian noise (AWGN) at relay $k$. During the second phase, each relay simultaneously retransmits the signal:

$$
x_{k}=a_{k} w_{k} y_{k}
$$

where $w_{k}$ denotes the beamforming weight for relay $k$ to be optimized. When the normalized amplifying gain is considered as

$$
a_{k}=\frac{1}{\sqrt{P_{s}\left|h_{s, k}\right|^{2}+\sum_{i=1}^{I} P_{i}\left|g_{i, k}\right|^{2}+1}},
$$

the transmission power of $x_{k}$ becomes $\left|w_{k}\right|^{2}$.

Aggregate transmit power over all relays is assumed to be constrained by $\sum_{k=1}^{K}\left|w_{k}\right|^{2} \leq P_{r}$, where $P_{r}$ is the maximum transmission power available at each relay. The assumption makes the DBF more practical at the network point of view. With the constraint, the total used power remains constant regardless of the number of relays $K$. It is an effective way to constrain the interference to other nodes in the network. Moreover, under the assumption, the transmission power cannot be shared among different nodes, which may not be practical. The received signal at $d$ is given by

$$
y_{d}=\sum_{k=1}^{K} h_{k, d} a_{k} w_{k} y_{k}+\sum_{i=1}^{I} g_{i, d} \sqrt{P_{i}} x_{i, 2}+n_{d}
$$

where $n_{d}$ is complex AWGN and $x_{i, 2}$ 's are the interfering signals during the second phase with powers $P_{i}$ 's. It is assumed that $E\left[\left|x_{j}\right|^{2}\right]=1$, where $x_{j} \in\left\{x_{s}, x_{i, 1}, x_{i, 2}\right\}$. 
Using a beamforming weight vector $\mathbf{w}=\left[w_{1}^{*}, \ldots, w_{K}^{*}\right]$, the SINR of the received signal at $d$ is represented by

$$
\begin{aligned}
& \gamma_{d} \\
& =\frac{P_{s} \mathbf{w a}^{H} \mathbf{a w}{ }^{H}}{\mathbf{w A}^{H} \mathbf{B} \mathbf{A} \mathbf{w}^{H}+\mathbf{w} \mathbf{A}^{H} E\left[\mathbf{n}^{H} \mathbf{n}\right] \mathbf{A} \mathbf{w}^{H}+\sum_{i=1}^{I} P_{i}\left|g_{i, d}\right|^{2}+1},
\end{aligned}
$$

where

$$
\begin{aligned}
\mathbf{a} & =\left[a_{1} h_{s, 1} h_{1, d}, \ldots, a_{K} h_{s, K} h_{K, d}\right], \\
\mathbf{A} & =\operatorname{diag}\left[a_{1} h_{1, d}, \ldots, a_{K} h_{K, d}\right], \\
\mathbf{B} & =\left[\begin{array}{ccc}
\sum_{i=1}^{I} P_{i}\left|g_{i, 1}\right|^{2} & \sum_{i=1}^{I} P_{i} g_{i, 1}^{*} g_{i, 2} & \sum_{i=1}^{I} P_{i} g_{i, 1}^{*} g_{i, K} \\
\sum_{i=1}^{I} P_{i} g_{i, 2}^{*} g_{i, 1} & \sum_{i=1}^{I} P_{i}\left|g_{i, 2}\right|^{2} & \vdots \\
\vdots & \ddots & \\
\sum_{i=1}^{I} P_{i} g_{i, K}^{*} g_{i, 1} & \ldots & \sum_{i=1}^{I} P_{i}\left|g_{i, K}\right|^{2}
\end{array}\right], \\
\mathbf{n} & {\left[\begin{array}{lll}
\left.n_{1}, \ldots, n_{K}\right] .
\end{array}\right.}
\end{aligned}
$$

\section{Distributed Beamforming with CCI Based on Local CSI}

Fact 1. When $\mathbf{T}$ is positive definite Hermitian, the following modified Rayleigh-Ritz theorem holds for any row vector $\mathbf{x}$ [2, Proposition 1]:

$$
\frac{\mathbf{x s}^{H} \mathbf{s x}}{\mathbf{x} \mathbf{T x}^{H}} \leq \lambda_{\text {max }},
$$

where $\lambda_{\max }=\operatorname{Tr}\left[\left(\mathbf{T}^{H / 2}\right)^{-1}\left(\mathbf{s}^{H} \mathbf{s}\right)\left(\mathbf{T}^{1 / 2}\right)^{-1}\right]$ is the largest eigenvalue of $\left(\mathbf{T}^{H / 2}\right)^{-1}\left(\mathbf{s}^{H} \mathbf{s}\right)\left(\mathbf{T}^{1 / 2}\right)^{-1}$ and the equality holds when $\mathbf{x}=c \mathbf{s} \mathbf{T}^{-1}$ for any nonzero constant $c$.

When there is no limit on available CSI at each relay, that is, global CSI is available, the optimal beamforming weight vector $\mathbf{w}_{g c}$ that maximizes the received SINR $\gamma_{d}$ in (5) is given by

$$
\mathbf{w}_{g c}=\mathbf{a V}^{-1} \sqrt{\frac{P_{r}}{\left\|\mathbf{a} \mathbf{V}^{-1}\right\|^{2}}},
$$

where

$$
\mathbf{V}=\mathbf{A}^{H} \mathbf{B A}+\mathbf{A}^{H} \mathbf{A}+\frac{\sum_{i=1}^{I} P_{\mathrm{i}}\left|g_{i, d}\right|^{2}+1}{P_{r}} \mathbf{I}_{K} .
$$

The proof is as follows. The received SINR $\gamma_{d}$ in (5) becomes

$$
\begin{aligned}
\gamma_{d} & =P_{s} \\
\cdot & \frac{\mathbf{w a}^{H} \mathbf{a w}^{H}}{\mathbf{w}\left[\mathbf{A}^{H} \mathbf{B A}+\mathbf{A}^{H} \mathbf{A}+\left(\left(\sum_{i=1}^{I} P_{i}\left|g_{i, d}\right|^{2}+1\right) / P_{r}\right) \mathbf{I}_{K}\right] \mathbf{w}^{H}} \\
& =P_{s} \frac{\mathbf{w a}^{H} \mathbf{a w}^{H}}{\mathbf{w} \mathbf{V} \mathbf{w}^{H}},
\end{aligned}
$$

where $E\left[\mathbf{n}^{H} \mathbf{n}\right]=\mathbf{I}_{K}$. From Fact 1, the optimal vector $\mathbf{w}_{g c}$ in (8) is obtained, where the value of $c=\sqrt{P_{r} /\left\|\mathbf{a V}^{-1}\right\|^{2}}$ is chosen to meet the aggregate the power constraint $P_{r}$.

However, using $\mathbf{w}_{g c}$ is not realistic for DBF. To calculate $\mathbf{w}_{g c}$ in a distributed way, $\mathbf{V}$ should be delivered to each relay, but it requires a significant burden because (1) acquiring $\mathbf{V}$ causes very high complexity since all the individual channel coefficients of interference channel $g_{m, n}$ 's must be estimated and (2) sharing $\mathbf{V}$ causes large overhead. Therefore, using $\mathbf{w}_{g c}$ in DBF is impractical, especially when $K$ or $I$ is large. To mitigate this problem, the following theorem introduces a simple DBF when only local CSI is available at each relay.

Theorem 1. When only local CSI is available at each relay, the optimal beamforming weight vector $\mathbf{w}_{l c}$ that maximizes the received SINR $\gamma_{d}$ is given by

$$
\mathbf{w}_{l c}=\mathbf{u} \sqrt{\frac{P_{r}}{\|\mathbf{u}\|^{2}}}
$$

where $\mathbf{u}$ is

$$
\begin{aligned}
\mathbf{u}= & {\left[\frac{h_{s, 1} h_{1, d} / a_{1}}{P_{r}\left|h_{1, d}\right|^{2}\left(\sum_{i=1}^{I} P_{i}\left|g_{i, 1}\right|^{2}+1\right)+\left(\sum_{i=1}^{I} P_{i}\left|g_{i, d}\right|^{2}+1\right) / a_{1}^{2}},\right.} \\
& \ldots, \\
& \left.\frac{h_{s, K} h_{K, d} / a_{K}}{P_{r}\left|h_{K, d}\right|^{2}\left(\sum_{i=1}^{I} P_{i}\left|g_{i, K}\right|^{2}+1\right)+\left(\sum_{i=1}^{I} P_{i}\left|g_{i, d}\right|^{2}+1\right) / a_{K}^{2}}\right] .
\end{aligned}
$$

Proof. To calculate the weight coefficient $w_{k}$ at each relay with only local CSI, V in (9) must be a diagonal matrix, and relay $k$ needs to be able to estimate $[\mathbf{V}]_{k, k}$ without communication between relays. Therefore, $\mathbf{B}$ must be replaced by

$$
\widetilde{\mathbf{B}}=\operatorname{diag}\left[\sum_{i=1}^{I} P_{i}\left|g_{i, 1}\right|^{2}, \ldots, \sum_{i=1}^{I} P_{i}\left|g_{i, K}\right|^{2}\right]
$$

From Fact $1, \mathbf{w}_{l c}$ is obtained by

$$
\mathbf{w}_{l c}=\mathbf{a} \widetilde{\mathbf{V}}^{-1} \sqrt{\frac{P_{r}}{\left\|\mathbf{a} \widetilde{\mathbf{V}}^{-1}\right\|^{2}}},
$$

where

$$
\widetilde{\mathbf{V}}=\mathbf{A}^{H} \widetilde{\mathbf{B}} \mathbf{A}+\mathbf{A}^{H} \mathbf{A}+\frac{\sum_{i=1}^{I} P_{i}\left|g_{i, d}\right|^{2}+1}{P_{r}} \mathbf{I}_{K} \cdot
$$


Because $\widetilde{\mathbf{V}}$ is a diagonal matrix, its inverse is easily obtained from $\left[\widetilde{\mathbf{V}}^{-1}\right]_{k, k}=1 /[\widetilde{\mathbf{V}}]_{k, k}$, and closed-form $\mathbf{w}_{l c}$ is obtained as in (11) and (12).

Each relay calculates $w_{l c, k}$ in a distributed way with only local CSI $h_{s, k}, h_{k, d}$, and $\sum_{i=1}^{I} P_{i}\left|g_{i, k}\right|^{2}$ when $\left(\sum_{i=1}^{I} P_{i}\left|g_{i, d}\right|^{2}+1\right)$ and $\|\mathbf{u}\|^{2}$ are delivered from the destination (to calculate $\|\mathbf{u}\|^{2}$ with very small overhead, several methods are available such as training-sequence-based channel estimation [5-7]). In this sense, $\mathbf{w}_{l c}$ is called a DBF vector with local CSI. Therefore, $\mathbf{w}_{l c}$ induces very small overhead. Moreover, calculating $\mathbf{w}_{l c}$ causes low complexity, because each relay estimates not $g_{m, n}$ 's but corresponding aggregate interference plus noise power $\left(\sum_{i=1}^{I} P_{i}\left|g_{i, k}\right|^{2}+1\right)$, which is much easier to estimate $[8,9]$. Nevertheless, $\mathbf{w}_{l c}$ still shows excellent performance as follows: (1) $\mathbf{w}_{l c}$ achieves nearly the optimum performance of $\mathbf{w}_{g c}$ when $I$ is large enough or interference power toward relays is small and (2) $\mathbf{w}_{l c}$ achieves the capacity scaling of $(1 / 2) \log K$, which corresponds to the maximal capacity scaling of cooperative relaying without CCI.

Corollary 2. When the number of interferers I is sufficiently large, it becomes $\widetilde{\mathbf{V}} \sim \mathbf{V}$, and, therefore, $\mathbf{w}_{l c}$ achieves the optimum performance of $\mathbf{w}_{g c}$.

Proof. Let $\mu_{k} \triangleq(1 / I) \sum_{i=1}^{I} E\left[P_{i}\left|g_{i, k}\right|^{2}\right]$. When $P_{i}$ is limited and $I \rightarrow \infty, a_{m} a_{n} \sim\left(1 / \sqrt{\mu_{m}} \sqrt{\mu_{n}}\right)(1 / I)$ and
$(1 / I) \sum_{i=1}^{I} P_{i} g_{i, m}^{*} g_{i, n} \sim\left\{0\right.$, for $m \neq n ; \mu_{m}$, for $\left.m=n\right\}$. Therefore,

$$
\begin{aligned}
{\left[\mathbf{A}^{H} \widetilde{\mathbf{B}} \mathbf{A}\right]_{m, n} } & =a_{m} a_{n} h_{m, d}^{*} h_{n, d} \sum_{i=1}^{I} P_{i} g_{i, m}^{*} g_{i, n} \\
& \sim \begin{cases}0, & \text { for } m \neq n \\
\left|h_{m, d}\right|^{2}, & \text { for } m=n,\end{cases}
\end{aligned}
$$

and $\widetilde{\mathbf{V}} \sim \mathbf{V}$.

When interference power toward relays is small, it is obvious that $\widetilde{\mathbf{V}} \approx \mathbf{V}$, and $\mathbf{w}_{l c}$ closely achieves the performance of $\mathbf{w}_{g c}$.

Theorem 3. When $K \rightarrow \infty$ with any finite $P_{s}, P_{r}$, and $P_{i}$, the ergodic capacity with $\mathbf{w}_{l c}, \bar{C}\left(\mathbf{w}_{l c}\right) \triangleq E\left\{C\left(\mathbf{w}_{l c}\right)\right\}$ converges to $(1 / 2) \log K$.

Proof. With $\mathbf{w}_{l c}$, the received SINR at $d$ becomes

$$
\begin{aligned}
& \gamma_{d}=P_{s} \frac{\mathbf{w}_{l c} \mathbf{a}^{H} \mathbf{a} \mathbf{w}_{l c}{ }^{H}}{\mathbf{w}_{l c} \mathbf{V} \mathbf{w}_{l c}{ }^{H}}=P_{s} \frac{\mathbf{w}_{l c} \mathbf{a}^{H} \mathbf{a} \mathbf{w}_{l c}{ }^{H}}{\mathbf{w}_{l c} \widetilde{\mathbf{V}} \mathbf{w}_{l c}{ }^{H}} \cdot \frac{\mathbf{w}_{l c} \widetilde{\mathbf{V}} \mathbf{w}_{l c}{ }^{H}}{\mathbf{w}_{l c} \mathbf{V} \mathbf{w}_{l c}{ }^{H}} \\
& \stackrel{(a)}{\sim} P_{s} \frac{\mathbf{w}_{l c} \mathbf{a}^{H} \mathbf{a} \mathbf{w}_{l c}{ }^{H}}{\mathbf{w}_{l c} \widetilde{\mathbf{V}} \mathbf{w}_{l c}{ }^{H}}=\sum_{k=1}^{K} N_{k},
\end{aligned}
$$

where

$$
N_{k}=\frac{\left(P_{s}\left|h_{s, k}\right|^{2} /\left(\sum_{i=1}^{I} P_{i}\left|g_{i, k}\right|^{2}+1\right)\right)\left(P_{r}\left|h_{k, d}\right|^{2} /\left(\sum_{i=1}^{I} P_{i}\left|g_{i, d}\right|^{2}+1\right)\right)}{P_{s}\left|h_{s, k}\right|^{2} /\left(\sum_{i=1}^{I} P_{i}\left|g_{i, k}\right|^{2}+1\right)+P_{r}\left|h_{k, d}\right|^{2} /\left(\sum_{i=1}^{I} P_{i}\left|g_{i, d}\right|^{2}+1\right)+1},
$$

and (a) follows from the fact that $\mathbf{w}_{l c} \widetilde{\mathbf{V}} \mathbf{w}_{l c}{ }^{H} / \mathbf{w}_{l c} \mathbf{V} \mathbf{w}_{l c}{ }^{H} \sim 1$ for sufficiently large $I$. The ergodic capacity with $\mathbf{w}_{l c}$ is given by [10]:

$$
\bar{C}\left(\mathbf{w}_{l c}\right) \sim E\left[\frac{1}{2} \log _{2}\left(1+\sum_{k=1}^{K} N_{k}\right)\right]
$$

where the factor $1 / 2$ denotes the rate loss because of the half-duplex constraint of relays. Because $N_{k}$ satisfies the Kolmogorov conditions as shown in the Appendix, the following theorem can be applied [11, Theorem 1.8.D]:

$$
\sum_{k=1}^{K} \frac{N_{k}}{K}-\sum_{k=1}^{K} \frac{E\left[N_{k}\right]}{K} \stackrel{\text { w.p. } 1}{\longrightarrow} 0
$$

Therefore, $\sum_{k=1}^{K} N_{K} \stackrel{\text { w.p. } 1}{\longrightarrow} \sum_{k=1}^{K} E\left[N_{k}\right]$, and $\bar{C}\left(\mathbf{w}_{l c}\right) \sim$ $(1 / 2) \log K$.

\section{Numerical Results}

In this section, $\bar{C}\left(\mathbf{w}_{l c}\right)$ is compared with $\bar{C}\left(\mathbf{w}_{c v}\right)$, where $\mathbf{w}_{c v}$ is the weight vector of a conventional DBF that maximizes the received SNR when there is no CCI [2]:

$$
\mathbf{w}_{c v}=\mathbf{v} \sqrt{\frac{P_{r}}{\|\mathbf{v}\|^{2}}}
$$

where

$$
\mathbf{v}=\left[\frac{h_{s, 1} h_{1, d} a_{1}}{1+a_{1}^{2} P_{r}\left|h_{1, d}\right|^{2}}, \ldots, \frac{h_{s, K} h_{K, d} a_{K}}{1+a_{K}^{2} P_{r}\left|h_{K, d}\right|^{2}}\right] .
$$

Comparing with $\mathbf{w}_{c v}, \mathbf{w}_{l c}$ requires only a slight increase in overhead and complexity in order to estimate $\sum_{i=1}^{I} P_{i}\left|g_{i, k}\right|^{2}+1$ at the corresponding relay and to feed back $\sum_{i=1}^{I} P_{i}\left|g_{i, d}\right|^{2}+$ 1 from the destination. It is assumed that the relays are located in the middle of the source and the destination, and, therefore, $\sigma_{s, k}^{2}=\sigma_{k, d}^{2}=1$, for all $k$. For comparison purposes, simulation results for $\bar{C}\left(\mathbf{w}_{g c}\right)$ are also plotted. According to the location of interferers, three cases are considered as follows. 


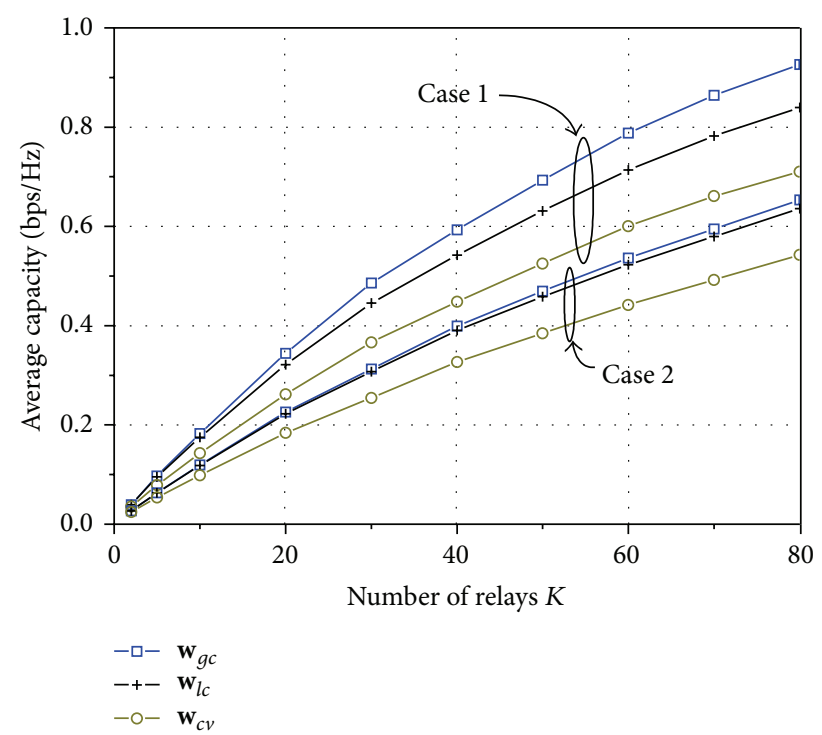

Figure 2: Comparison of ergodic capacity: $I=5, P_{s}=P_{r}=P_{i}=$ $10 \mathrm{~dB}$, and $\sigma_{s, k}^{2}=\sigma_{k, d}^{2}=1$, for all $k$ : Case 1 with $\sigma_{I, i, k}^{2}=1$ and $\sigma_{I, i, d}^{2}=1$ and Case 2 with $\sigma_{I, i, k}^{2}=0.5$ and $\sigma_{I, i, d}^{2}=3$.

Case 1. The distances between relays-interferers and destination-interferers are the same, and, therefore, the relays and the destination are affected by the same average interfering power with $\sigma_{I, i, k}^{2}=1$ and $\sigma_{I, i, d}^{2}=1$.

Case 2. The interferers are closely located to the destination with $\sigma_{I, i, k}^{2}=0.5$ and $\sigma_{I, i, d}^{2}=3$.

Case 3. The interferers are closely located to the relays with $\sigma_{I, i, k}^{2}=3$ and $\sigma_{I, i, d}^{2}=0.5$.

Figure 2 plots the ergodic capacity for Cases 1 and 2, and Figure 3 for Case 3, with parameter values of $I=5$ and $P_{s}=$ $P_{r}=P_{i}=10 \mathrm{~dB}$. For all cases, the figures show that $\bar{C}\left(\mathbf{w}_{g c}\right)>$ $\bar{C}\left(\mathbf{w}_{l c}\right)>\bar{C}\left(\mathbf{w}_{c v}\right)$ and $\mathbf{w}_{l c}$ achieves remarkable performance gains over $\mathbf{w}_{c v}$; when $K=40,21 \%, 20 \%$, and $29 \%$ gains are obtained for Cases 1, 2, and 3, respectively. Moreover, $\mathbf{w}_{l c}$ closely achieves $\bar{C}\left(\mathbf{w}_{g c}\right)$ for Case 2 because interference power toward relays is small, but $\mathbf{w}_{g c}$ is superior to $\mathbf{w}_{l c}$ for Cases 1 and 3 at the cost of greatly increased overhead and complexity.

As $I$ increases, however, $\mathbf{w}_{l c}$ closely achieves $\bar{C}\left(\mathbf{w}_{g c}\right)$ for all cases as shown in Figures 4 and 5, in which the ergodic capacity is plotted for $I=30, P_{s}=P_{r}=10 \mathrm{~dB}$, and $P_{i}=5 \mathrm{~dB}$. The figures shows that $\mathbf{w}_{l c}$ achieves nearly $\bar{C}\left(\mathbf{w}_{g c}\right)$ for all cases and also represents remarkable performance gains over $\mathbf{w}_{c v}$, greater than $21 \%$ for all cases when $K=40$.

\section{Conclusions}

This paper has proposed the optimal AF DBF $\mathbf{w}_{l c}$ in the presence of CCI when only local CSI is available at each relay. With slight increased overhead and complexity, $\mathbf{w}_{l c}$ efficiently reduces the impact of CCI and yields significant improvements over $\mathbf{w}_{c v}$. Using $\mathbf{w}_{l c}$ is more attractive when interference power toward relays is small or there are a large

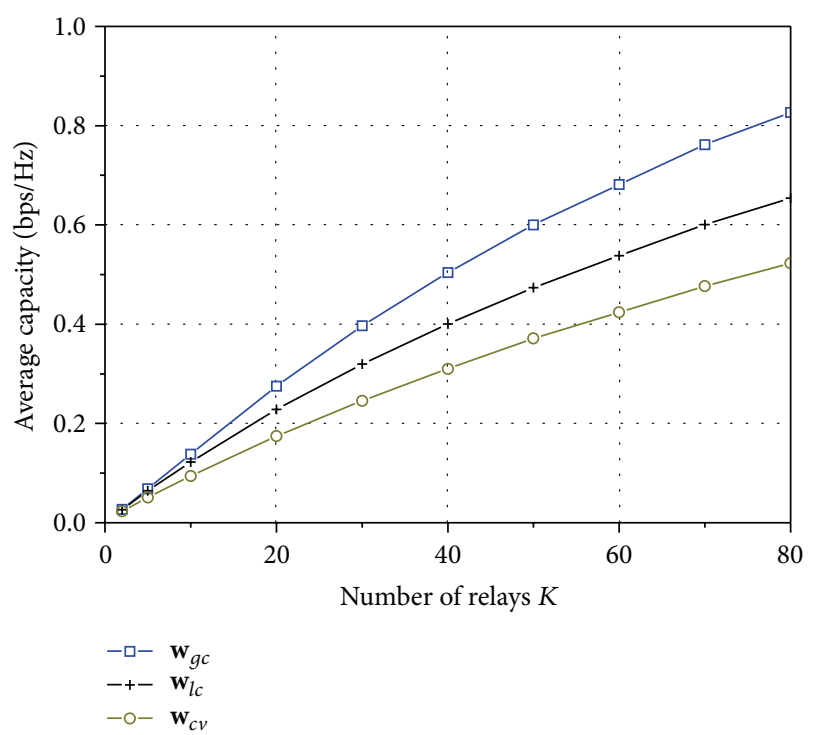

Figure 3: Comparison of ergodic capacity: $I=5, P_{s}=P_{r}=P_{i}=$ $10 \mathrm{~dB}$, and $\sigma_{s, k}^{2}=\sigma_{k, d}^{2}=1$, for all $k$ : Case 3 with $\sigma_{I, i, k}^{2}=3$ and $\sigma_{I, i, d}^{2}=$ 0.5 .

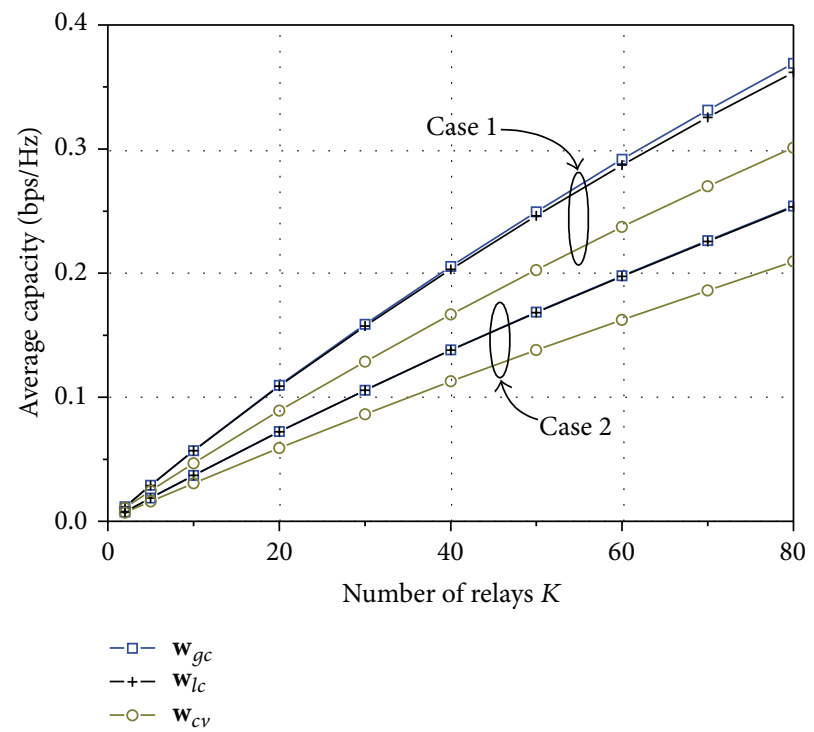

FIGURE 4: Comparison of ergodic capacity: $I=30, P_{s}=P_{r}=10 \mathrm{~dB}$, $P_{i}=5 \mathrm{~dB}$, and $\sigma_{s, k}^{2}=\sigma_{k, d}^{2}=1$, for all $k$ : Case 1 with $\sigma_{I, i, k}^{2}=1$ and $\sigma_{I, i, d}^{2}=1$, and Case 2 with $\sigma_{I, i, k}^{2}=0.5$ and $\sigma_{I, i, d}^{2}=3$.

number of interferers where $\mathbf{w}_{l c}$ achieves nearly the same performance as $\mathbf{w}_{g c}$.

\section{Appendix}

Lemma A.1. For any finite $P_{s}, P_{r}$, and $P_{i}$ with large $K, N_{k}$ in (18) satisfies the Kolmogorov conditions:

$$
\begin{aligned}
\sum_{k=1}^{K} \frac{\operatorname{VAR}\left[N_{k}\right]}{k^{2}} & <\infty, \\
\mu & \triangleq \frac{1}{K} \sum_{k=1}^{K} E\left[N_{k}\right]<\infty .
\end{aligned}
$$




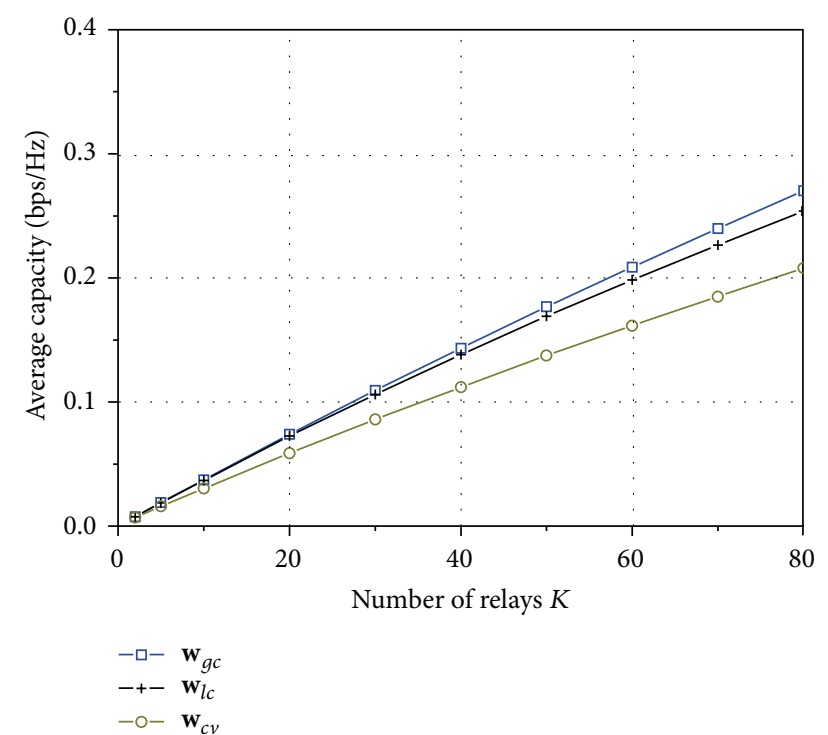

FIgURE 5: Comparison of ergodic capacity: $I=30, P_{s}=P_{r}=10 \mathrm{~dB}$, $P_{i}=5 \mathrm{~dB}$, and $\sigma_{s, k}^{2}=\sigma_{k, d}^{2}=1$, for all $k$ : Case 3 with $\sigma_{I, i, k}^{2}=3$ and $\sigma_{I, i, d}^{2}=0.5$.

Proof. Let $M_{k} \triangleq P_{s}\left|h_{s, k}\right|^{2} P_{r}\left|h_{k, d}\right|^{2} /\left(P_{s}\left|h_{s, k}\right|^{2}+P_{r}\left|h_{k, d}\right|^{2}+\right.$ 1). Then, $M_{k}<P_{s}\left|h_{s, k}\right|^{2} P_{r}\left|h_{k, d}\right|^{2} /\left(P_{s}\left|h_{s, k}\right|^{2}+P_{r}\left|h_{k, d}\right|^{2}\right)<$ $\min \left\{P_{s}\left|h_{s, k}\right|^{2}, P_{r}\left|h_{k, d}\right|^{2}\right\}<P_{s}\left|h_{s, k}\right|^{2}$. $P_{s}\left|h_{s, k}\right|^{2}$ 's are exponentially distributed r.v.s which mean $P_{s} \sigma_{s, k}^{2}$ and variance $\left(P_{s} \sigma_{s, k}^{2}\right)^{2}$ are bounded. Therefore, the Kolmogorov conditions $\sum_{k=1}^{K} \operatorname{VAR}\left[P_{s}\left|h_{s, k}\right|^{2}\right] / k^{2}<\infty$ and $(1 / K) \sum_{k=1}^{K} E\left[P_{s}\left|h_{s, k}\right|^{2}\right]<$ $\infty$ are satisfied. Since $N_{k}<M_{k}<P_{s}\left|h_{s, k}\right|^{2}, N_{k}$ also satisfies the Kolmogorov conditions.

\section{Conflict of Interests}

The authors declare that there is no conflict of interests regarding the publication of this paper.

\section{Acknowledgments}

This work was partly supported by National Research Foundation of Korea (NRF) grant funded by the Korea government (MSIP) (NRF-2015R1A2A2A01008218), Institute for Information \& Communications Technology Promotion (IITP) grant funded by the Korea government (MSIP) (no. B010115-0557, Resilient Cyber-Physical Systems Research), and the Robot Industry Fusion Core Technology Development Project of the Ministry of Trade, Industry \& Energy of Korea (10052980).

\section{References}

[1] H. Bölcskei, R. U. Nabar, Ö. Oyman, and A. J. Paulraj, "Capacity scaling laws in MIMO relay networks," IEEE Transactions on Wireless Communications, vol. 5, no. 6, pp. 1433-1444, 2006.

[2] Z. Yi and I.-M. Kim, "Joint optimization of relay-precoders and decoders with partial channel side information in cooperative networks," IEEE Journal on Selected Areas in Communications, vol. 25, no. 2, pp. 447-458, 2007.

[3] J.-B. Kim, I.-H. Lee, D. Kim, and J. Lim, "Asymptotic performances of near-optimal selective distributed beamforming for two-way relaying with partial channel state information," IEEE Communications Letters, vol. 15, no. 5, pp. 512-514, 2011.

[4] L. Yang and M.-S. Alouini, "Outage probability of distributed beamforming with co-channel interference," IEEE Communications Letters, vol. 16, no. 3, pp. 334-337, 2012.

[5] F. Gao, T. Cui, and A. Nallanathan, "On channel estimation and optimal training design for amplify and forward relay networks," IEEE Transactions on Wireless Communications, vol. 7, no. 5, pp. 1907-1916, 2008.

[6] N. Ahmed, M. A. Khojastepour, A. Sabharwal, and B. Aazhang, "Outage minimization with limited feedback for the fading relay channel," IEEE Transactions on Communications, vol. 54, no. 4, pp. 659-669, 2006.

[7] J.-B. Kim, J.-W. Choi, and J. M. Cioffi, "Cooperative distributed beamforming with outdated CSI and channel estimation errors," IEEE Transactions on Communications, vol. 62, no. 12, pp. 4269-4280, 2014.

[8] F. F. Digham, M.-S. Alouini, and M. K. Simon, "On the energy detection of unknown signals over fading channels," IEEE Transactions on Communications, vol. 55, no. 1, pp. 21-24, 2007.

[9] J.-B. Kim and D. Kim, "Outage probability and achievable diversity order of opportunistic relaying in cognitive secondary radio networks," IEEE Transactions on Communications, vol. 60, no. 9, pp. 2456-2466, 2012.

[10] J. N. Laneman and G. W. Wornell, "Distributed space-time coded protocols for exploiting cooperative diversity in wireless networks," IEEE Transactions on Information Theory, vol. 49, no. 10, pp. 2415-2425, 2003.

[11] R. J. Serfling, Approximation Theorems of Mathematical Statistics, John Wiley \& Sons, New York, NY, USA, 1980. 


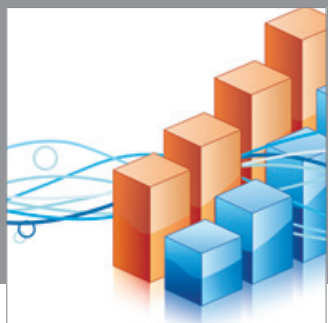

Advances in

Operations Research

mansans

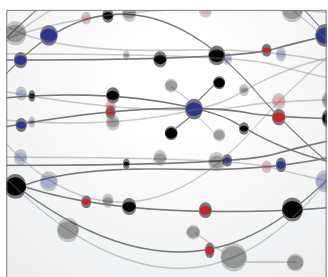

The Scientific World Journal
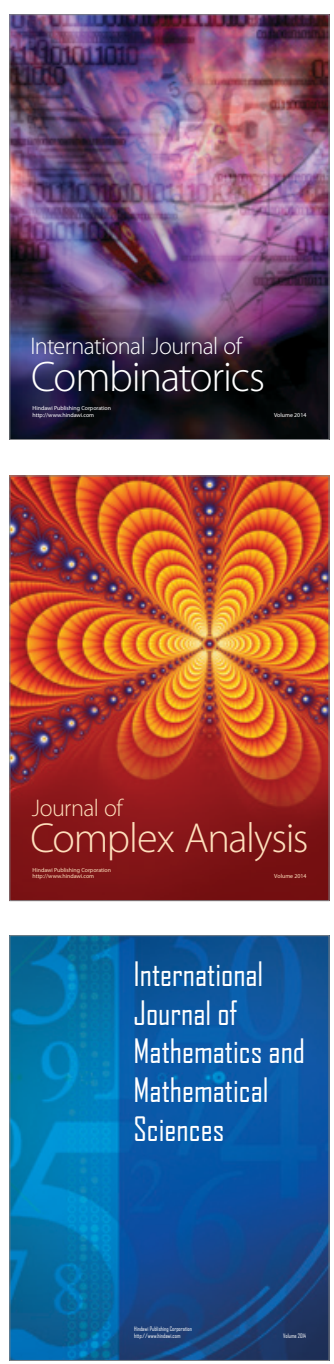
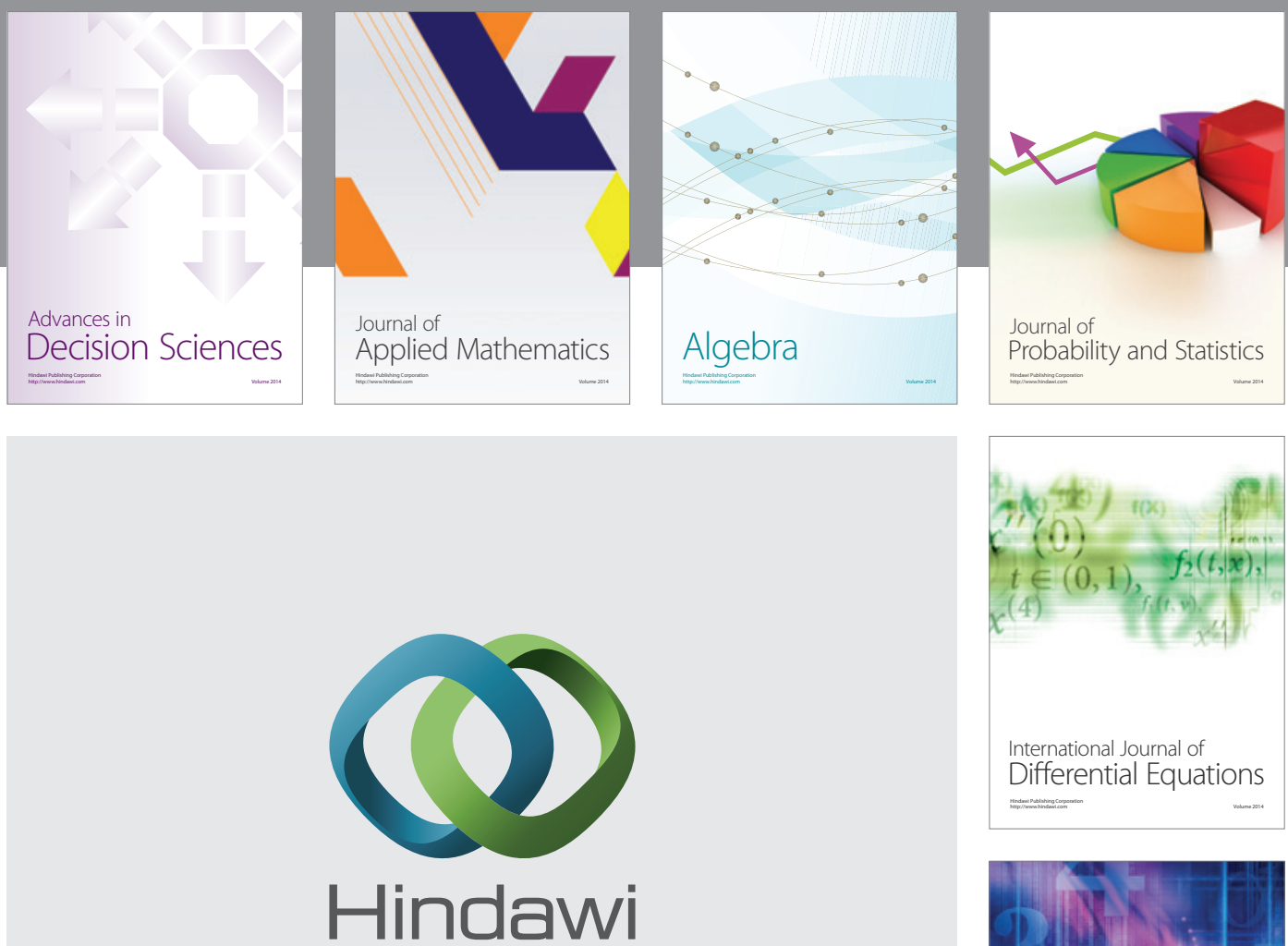

Submit your manuscripts at http://www.hindawi.com
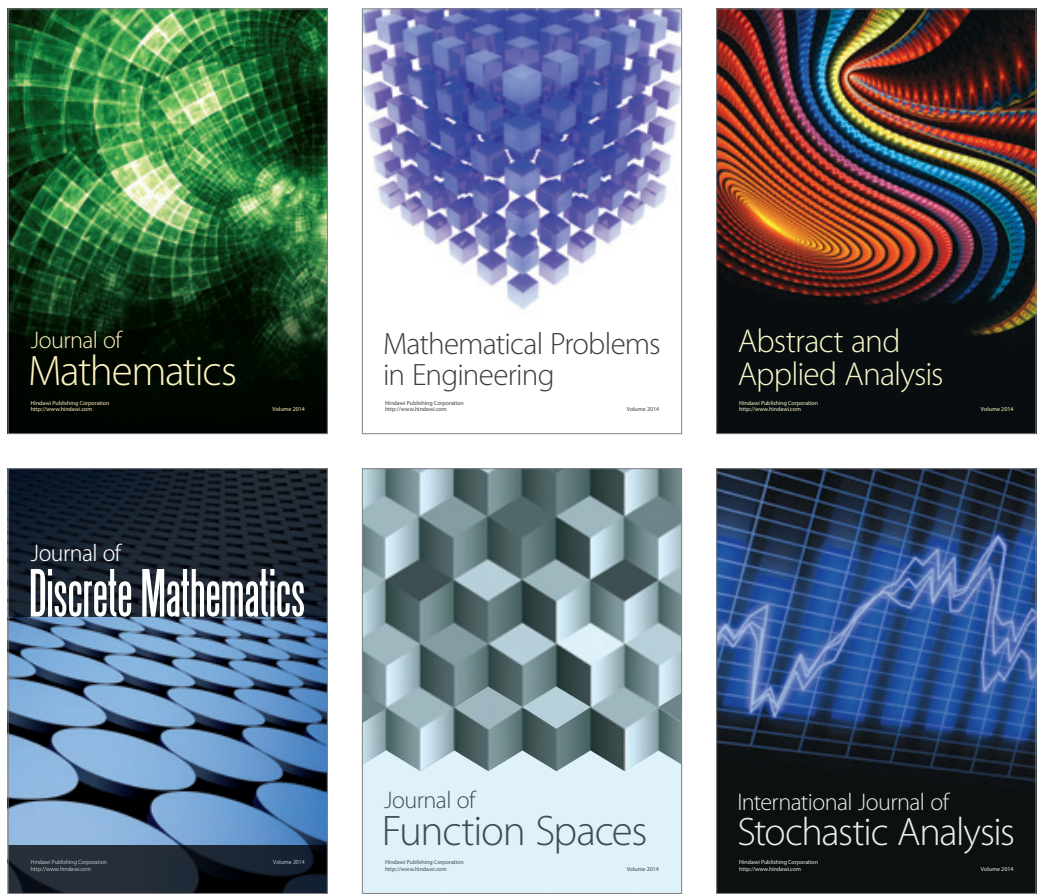

Journal of

Function Spaces

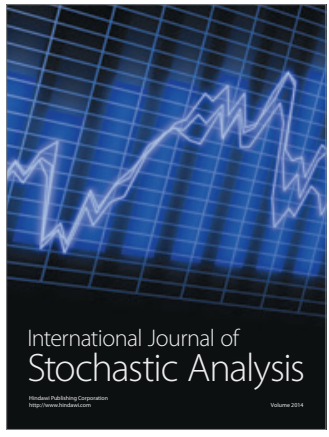

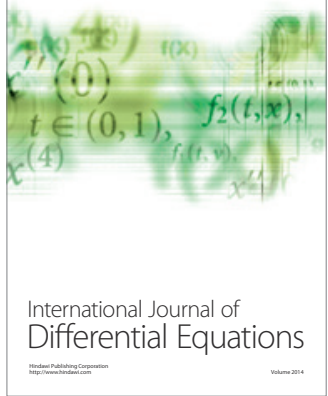
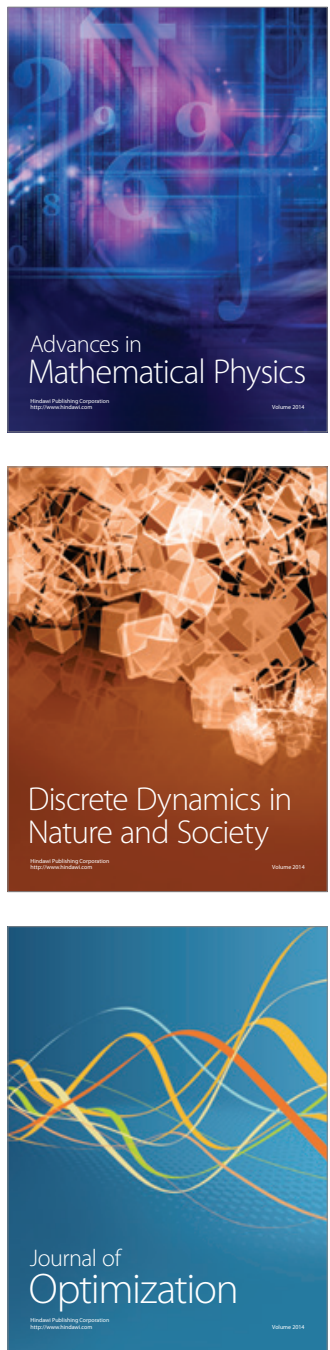